\title{
Im Spannungsfeld von Politik und Verfassung
}

\section{Zum Tod von Jürgen Seifert (I928-2005)}

Nach einem Vortrag an der Universität Münster Mitte der soer Jahre berichtete Wolfgang Abendroth, Widerstandskämpfer im Dritten Reich, demokratischer Staatsrechtler der ersten Stunde, dass er ganz unerwartet einen jungen linken Juristen kennen gelernt habe: Jürgen Seifert. In Münster studierte Seifert Rechtswissenschaft und Philosophie.1954 in die IG Metall und 1957 in die SPD eingetreten, gehörte er dem Sozialistischen Deutschen Studentenbund (SDS) an. Seiferts lebensgeschichtliche Prägung bestand darin, dass er, der Mitglied der Hitlerjugend war, der »Goebbels Wochenzeitung >Das Reich geradezu verschlungen« hat, wie er selber schreibt, nach 1945 den Bruch mit dem Nationalsozialismus in einem längeren Prozess mit großer Entschiedenheit vollzogen hat. Er wird zunächst Werkzeugmacher und erfährt die Welt der abhängigen Arbeit. In der Bücherei der IG Metall in Osnabrück findet er die Literatur, die sein politisches Denken zu formen beginnt.

Während die juristischen Legitimationshelfer der NS-Diktatur von Theodor Maunz bis Ulrich Scheuner, die die Beseitigung des Parlamentarismus, die rechtliche Schrankenlosigkeit der Führerdespotie und die Entrechtung der Juden gerechtfertigt hatten, die meisten rechtswissenschaftlichen Lehrstühle der jungen Republik besetzen, wird Seifert durch das Rechtsdenken derjenigen, die von den Nazis besiegt, verfolgt, inhaftiert und ins Exil getrieben wurden, in wesentlichem Maße beeinflusst. Dies sind vor allem die Theoretiker der Weimarer Sozialdemokratie wie Franz L. Neumann, Otto Kirchheimer, Arcadius Gurland und der oppositionelle Kommunist Karl Korsch. Wolfgang Abendroth, der auf besondere Weise, etwa mit seiner Interpretation des Verfassungsbegriffs des demokratischen und sozialen Rechtsstaats, die Brücke zu der juristischen Linken der ersten Republik schlägt, wird für Seiferts Verständnis des Grundgesetzes besonders wichtig. Dies gilt vor allem für die Unterscheidung von Staatsapparat und Verfassung und für das Theorem, dass die demokratische Rechtsordnung als Rahmenregelung für die Austragung politischer und gesellschaftlicher Konflikte zu begreifen ist.

Die Aneignung des Denkens gesellschaftskritischer, der Gedankenwelt der Aufklärung verpflichteter Juristen verband sich bei Seifert mit der Rezeption des westlichen Marxismus, der im direkten Gegensatz zur Herrschaftsideologie des Ostblocks stand. Eingehend studierte er in einer fotokopierten Ausgabe Georg Lukács' Buch »Geschichte und Klassenbewusstsein « von I923, das die ökonomistische Verkürzung des Marxismus durch die Entfaltung des Totalitätsbegriffs zu überwinden sucht.

Während seines Studiums und in der Zeit danach entwickelt Seifert für längere Zeit eine persönliche, auch in einem Briefwechsel sich niederschlagende Beziehung zu Carl Schmitt, die bei allen Disputen von Achtung für Schmitt und von einer Genugtuung über die Anerkennung seiner Aphorismen durch sein Gegenüber gekennzeichnet ist. Als er aber auf Schmitts antisemitische Schmähschrift von 1936 stößt, die sich der Worte Hitlers zur Rechtfertigung der Erniedrigung der Juden bedient, vollzieht er einen absoluten Bruch, der sich am deutlichsten in seinem Nachruf auf den einflussreichsten Juristen des Dritten Reiches ausdrückt. Er zeigt, dass sich an Schmitts Begriffsbildung die Gefahren für eine rechtsstaatliche Demokratie besonders deutlich erkennen, lassen, denn seine 
Positionen und Begriffe zielen auf eine »machtstaatliche Entrechtlichung « jener Kräfte und Gruppen, die eine oppositionelle Haltung zur bürgerlichen Gesellschaft einnehmen.

Dass Seifert sich über viele Jahre mit Carl Schmitt beschäftigt, mag auch mit der weitgehend unbeantwortet gebliebenen Frage nach der Haltung seines Vaters im NS-System zusammenhängen. Dessen Rolle hatte Seifert so beschrieben: »Mein Vater ... war mitverantwortlich für die auf eine Notverordnung Hindenburgs gestützte Telefon- und Fernmeldeüberwachung von NS-Gegnern ..., die manchen Gegner des NS-Systems ins KZ oder in den Tod gebracht haben.« Diese Tätigkeit für das Regime wurde weitgehend mit Schweigen überdeckt. Um so eher bot sich ein anderer, maßgebender Repräsentant des autoritären Systems als Gesprächspartner für die intellektuelle Selbstfindung an.

Die Aneignung der theoretischen Instrumentarien eines Rechtsdenkens »aus Freiheitssinn « (Radbruch), wie es im Grundgesetz, der Gegenverfassung zur NS-Herrschaft, seinen Niederschlag gefunden hat, befähigten Seifert, I96 I aus der SPD wegen seiner Mitgliedschaft im SDS ausgeschlossen, in besonderer Weise als juristischer Berater und politischer Stratege, vor allem der IG Metall, den wichtigsten verfassungspolitischen Kampf in der Bundesrepublik, die Auseinandersetzung um die Notstandsgesetze, die die 6oer Jahre beherrschte, zu führen. In dieser Zeit schrieb Seifert sein in mehreren Auflagen verbreitetes Buch »Gefahr im Verzuge - zur Problematik der Notstandsgesetze« (1963). Zu dem Buch steuerte der sozialdemokratische hessische Generalstaatsanwalt Fritz Bauer, auf Seiferts Bitte, eine Einleitung bei, die nicht nur die politischen und rechtlichen Gefahren einer fessellosen Exekutivgewalt in den Blick rückte, sondern auch den Gewerkschaften demonstrierte, dass ein gerade aus der SPD ausgeschlossener Jurist wissenschaftlich persona grata blieb.

In der Auseinandersetzung mit den geplanten Regelungen für den Ausnahmezustand bildete sich bei Seifert die auch für spätere Arbeiten charakteristische Verbindung von wissenschaftlicher Argumentation, die den Anspruch auf rationale Stringenz stellt, und politischer Intervention heraus, die sich des Rückhalts bei gesellschaftlichen Kräften bzw. der öffentlichen Meinung zu versichern sucht. In Anlehnung an Karl Korsch nennt Seifert dies eine »juristische Aktion «, die nicht abstrakte Forderungen stellt, sondern auf die jeweiligen Entscheidungssituationen zielt.

Seiferts Position in der Notstandsauseinandersetzung richtete sich - vor allem gemeinsam mit Wolfgang Abendroth, Helmut Ridder, Karl Dietrich Bracher und Jürgen Habermas - gegen die Etablierung eines Ausnahmezustands, der zur »Stunde der Exekutive« werden sollte, wie Innenminister Schröder I96o forderte. Dann sind die Grundrechte, vor allem die Meinungsfreiheit und die Koalitionsfreiheit, ebenso zur Disposition gestellt wie die parlamentarischen Entscheidungs- und Kontrollkompetenzen. In einer Fülle von Expertisen, Reden und Aufsätzen, die auch in einem von ihm formulierten Alternativentwurf der SPD-Bundestagsabgeordneten Matthöfer, Gscheidle u. a. mündeten, besteht das Gewicht seiner Argumentation darin, dass er sie am juristischen Detail - und nicht allgemein polemisch - entwickelt und damit die Gegenseite in Begründungsschwierigkeiten bringt. Als I965 im Deutschen Bundestag zwischen der CDU/CSU und der SPD ein sogenannter Notstandskompromiss geschlossen wird, legt Seifert in einem für den Vorsitzenden der IG Metall verfassten Briefentwurf exakt dar, dass in der geplanten Regelung die Koalitionsund die Berufsfreiheit nicht unzweideutig geschützt seien. Die gewählte Formulierung, dass ^für $Z$ wecke der Verteidigung Dienstverpflichtungen « verfügt wer- 
den können, erlaube es, « das demokratische Grundrecht des Art.I 2 unter dem Vorwand eines Notstands einzuschränken «. Otto Brenner nahm die Argumentation von Seifert auf. Es kam zu einer breiten, von Intellektuellen unterstützten Aktionsfront der Gewerkschaften, die dazu führte, dass die SPD von der Vereinbarung mit der CDU wieder abrückte.

Die Notstandsverfassung von I968, Endpunkt des langjährigen Kampfes um die Struktur des Grundgesetzes, interpretierte Seifert, anders als Köpfe der Studentenbewegung wie Hans Jürgen Krahl, der von einem Ende der Demokratie sprach, als halbe Niederlage und als halben Sieg, weil auch rechtsstaatliche Sicherungen - durch die Erhaltung parlamentarischer Kompetenzen und der NichtEinschränkbarkeit zentraler Grundrechte - durchgesetzt wurden.

Seiferts Erfahrungen in der Notstandsauseinandersetzung bleiben für seine weitere wissenschaftliche und politische Rolle in der Bundesrepublik relevant. Nachdem er 197 I Professor für Politische Wissenschaft an der Universität Hannover geworden ist, wird er, zeitlich fast parallel, Bundesvorsitzender der Humanistischen Union und Mitglied der Redaktion der »Kritischen Justiz«. Wieder verbindet er verfassungsjuristische Analysen mit vielfachen Eingriffen in das politische Geschehen.

Er interveniert z. T. mit Erfolg gegen extra-legale, mit einem Rechtsschein versehene Operationen des Staatsapparats. Er kritisiert die Nichtachtung der Unverletzlichkeit der Wohnung des Atomwissenschaftlers Traube durch den Innenminister der Regierung Schmidt, analysiert die Auflösung grundrechtlicher Garantien für Bewerber für den öffentlichen Dienst, macht die Aushöhlung des Telefongeheimnisses durch die Nicht-Unterrichtung der von Abhörmaßnahmen Betroffenen öffentlich.

Seiferts Tätigkeit erschöpft sich nicht in rechtsstaatlich demokratisch angeleiteten Interventionen. Auf dem Feld der politischen und rechtlichen Theorie hat er eine Vielzahl von Arbeiten - über Hegel, Marx, Arendt, Abendroth und andere - vorgelegt, in denen die Fragen nach den unabdingbaren politischen und rechtlichen Formen eines bisher nur in Ansätzen verwirklichten Gemeinwesens umkreist werden, das den Krieg aus der Politik verbannt, die ökologischen Grundlagen der Menschheit sichert und die Selbstbestimmung der Individuen durch das rechtsstaatlich demokratische Regelwerk sichert.

Die angemessenen Formen politischen Konflikts, die in jeder Gesellschaft bestehen werden, sind Gegenstand einer programmatischen Abhandlung über die Strukturdifferenz von Feindschaft und Gegnerschaft. Dem Theorem Carl Schmitts, dass die in der physischen Vernichtung kulminierende Feindschaft den Begriff des Politischen ausmache, setzt er - auch in einem bemerkenswerten Exkurs über die biblische Geschichte von Kain und Abel - die in der Tradition der Aufklärung und der demokratischen Verfassungsurkunden sich ausdrükkende Position entgegen, dass sich Kontroversität allein in den Formen politischer Gegnerschaft, die durch die Freiheitsrechte ermöglicht und begrenzt wird, zu bewegen hat: »Freiheit gibt es nur dort, wo es gelingt, Feindschaft durch Gegnerschaft zu ersetzen.«

Der Redaktion der »Kritischen Justiz« gehörte Seifert 20 Jahre an. Schon am Gründungsprozess unserer Zeitschrift im Jahr 1968 beteiligt, publizierte er von Anbeginn kontinuierlich Aufsätze, Glossen, Rezensionen und Entgegnungen. Als Konflikte in der Redaktion über die kritische Ausrichtung des Blattes nicht auflösbar schienen und ein Auseinanderfallen der Redaktion drohte, baten wir es war 1978 - Seifert, in die Redaktion einzutreten. Damit konnten die Blocka- 
den aufgelöst werden. Die Kompromissfähigkeit, die Seifert als Umgangsmaxime in die Redaktion einbrachte, hat dazu nicht wenig beigetragen.

Für die Entwicklung seiner Positionen brauchte Seifert das Gespräch mit anderen, mit denen er kontrovers diskutieren konnte. Fast jeden seiner Texte veröffentlichte er erst, wenn sie durch das Purgatorium kritischen Gegenlesens, durch das sie eine andere Gestalt annahmen, gegangen waren. Im Umgang mit Kindern, die nicht durch das Realitätsprinzip bestimmt waren, hatte er spontane Freude. Für ihn war, auch bedingt durch den Gedanken, "alle Verhältnisse umzuwerfen, in denen der Mensch ein erniedrigtes...Wesen ist « (Marx), Hilfsbereitschaft etwas Selbstverständliches, die seinen Freunden, die unter seelischer Not litten, ebenso galt wie seiner Sekretärin, die Schwierigkeiten mit ihrem Vermieter hatte, oder Demonstranten, die einem rechtswidrigen Polizeieinsatz ausgesetzt waren. Seine moralische Sensibilität konnte urplötzlich in eine emotionale Explosion umschlagen, die ihm da und dort auch zu schaffen machte. Er rief dann, noch während seiner Attacken, abmildernd, dass man ihn doch kenne. Kurzum: Jürgen Seifert besaß in reichlichem Maße jenes humane Mitgefühl, das in der juristischen Tätigkeit oftmals zum Verschwinden gebracht wird. Bei ihm wirkte Empathie, zumal für die Schwächeren, als Antriebskraft seines Kampfes um Verfassungspositionen.

Ein Wort von Max Horkheimer gilt auch für Seifert: »Die Trauer (enthält) nicht bloß die Klage, sondern auch den Dank, dass der gelebt hat, den wir verloren haben. Es tut dem ..., der nicht mehr ist, ein Unrecht an, wenn etwas von dem Glück, das mit seiner Existenz für uns verbunden war, sich nicht auf sein Bild überträgt, als Kraft gegen den Schmerz.«

\section{Einige Arbeiten von Jürgen Seifert:}

- Kampf um Verfassungspositionen, Köln 1974

- Grundgesetz und Restauration, 3. Aufl., Neuwied i977

- Politik zwischen Destruktion und Gestaltung, Hannover 1997

- Der lange Weg, Hitler in mir zu überwinden. Antworten an meine Tochter Anna, Vorgänge H.r/r 984 , S. 34 ff. 